\title{
A CLASS OF FREE BOUNDARY PROBLEMS WITH ONSET OF A NEW PHASE
}

\author{
PATRICK GUIDOTTI *
}

\begin{abstract}
A class of diffusion driven Free Boundary Problems is considered which is characterized by the initial onset of a phase and by an explicit kinematic condition for the evolution of the free boundary. By a domain fixing change of variables it naturally leads to coupled systems comprised of a singular parabolic initial boundary value problem and a Hamilton-Jacobi equation. Even though the one dimensional case has been thoroughly investigated, results as basic as well-posedness and regularity have so far not been obtained for its higher dimensional counterpart. In this paper a recently developed regularity theory for abstract singular parabolic Cauchy problems is utilized to obtain the first well-posedness results for the Free Boundary Problems under consideration. The derivation of elliptic regularity results for the underlying static singular problems will play an important role.
\end{abstract}

Key words. Free Boundary Problem, Kinematic Condition, Singular Parabolic and Elliptic Equations, Well-posedness, Existence and Regularity.

AMS subject classifications. 35A07, 35C15, 35M10, 35J70, 35K65.

1. Introduction. In this paper we consider a class of one phase Free Boundary Problems (FBP) characterized by the initial onset of a phase. Such Free Boundary Problems arise in the description of diffusion in polymers for instance. Under physically natural conditions, these problems lead to a formulation in which the phase is initially absent. This feature manifests itself mathematically in that some of the equations in the nonlinear system become singular (if they are written in a fixed reference domain). In spite of the fact that this type of problems have been intensively studied in the literature over an extended period of time, all but one publication do not deal with the singular case considered here in more than one space dimension. The simplifying assumption that the phase be initially non-empty is typically added to avoid the mathematical complications stemming from the singularity. The one dimensional case has, however, been thoroughly investigated ([?, ?, ?, ?, ?, ?]) in the specific context of diffusion in polymers and, more generally, for diffusion driven FBPs ([?],[?] for instance). The methods used for the one dimensional case rely on the explicit use of the heat kernel to reduce the problem to the boundary. This approach cannot be used in higher dimensions because the singular behavior induced by the initial condition on the FBP can not be decoupled from the diffusion operator. This is due the the fact that the free boundary has a non trivial geometry in this case and to the fact that fundamental solutions (evolution operators) have not been studied for singular parabolic problems for which the singularity affects the underlying elliptic operator in an anisotropic way. The higher dimensional problem has recently been studied in [?] but only in its simpler quasi-stationary form. [?] established well-posedness for the problem in a class of functions which needs to be carefully crafted and leads to an asymptotic expansion for the singularity which is valid in the corresponding topologies and has practical relevance (cf. [?, ?]). Regularity results, maximal regularity in particular, for singular parabolic equations play a crucial role in this paper and have been recently obtained by the author in [?] where a construction of the evolution operator is given for a wide class of singular

\footnotetext{
*Department of Mathematics, University of California at Irvine, Irvine, CA 92697-3875 (gpatrick@math.uci.edu).
} 
parabolic problems. Maximal regularity is needed because the full problem will be solved by reduction to a model problem and perturbation about it. Since the ensuing perturbation is of maximal order (both in the singularity and differentiation) optimal regularity results are necessary. Previous abstract results were obtained by [?, ?], but they do not apply to the situation considered here because their validity does not cover the case of spaces of classical point-wise regularity, nor the case in which the singularity affects the equations anisotropically. Classical point-wise regularity is needed in the analysis proposed here since the singular parabolic equation is coupled to a Hamilton-Jacobi type equation modeling the front's dynamics. It has long been observed, as mentioned above, that one dimensional techniques can not be extended to the higher dimensional case. Summarizing, the presence of singular coefficients and the coupling to a Hamilton-Jacobi equations are two of the characterizing features of the problem under consideration. They make its analysis more difficult and delicate than that of the related but different classical Stefan-problem. This paper therefore offers a successful approach that fully overcomes these difficulties.

The unknowns of the problem are a function $u: \Omega_{t} \rightarrow \mathbb{R}$ defined on the open domain $\Omega_{t} \subset \mathbb{R}^{n+1} \ni(x, y)$ and its unknown boundary $\Gamma_{t}$. The function $u$ measures the concentration of the penetrant in the case of diffusion in polymers. Many geometries can be chosen for the domain $\Omega_{t}$. Here a strip-like setting is chosen where the domain is bounded by a fixed lower, and an upper moving hyper-surface denoted by $\Gamma_{0}$ and $\Gamma_{t}$, respectively. Other configurations, like annulus-type domains are possible and interesting and the results obtained here would apply to those since they all would lead to the same local model problems. The system of equations satisfied by $\left(u, \Gamma_{t}\right)$ proposed in [?] and generalizing [?, ?] then reads

$$
\begin{aligned}
\varepsilon u_{t}-\triangle_{x, y} u & =0, & & \text { in } \bigcup_{t>0}\{t\} \times \Omega_{t}, \\
u & =g, & & \text { on }(0, \infty) \times \Gamma_{0}, \\
-\partial_{\nu_{t}} u & =(1+\varepsilon u) V, & & \text { on } \bigcup_{t>0}\{t\} \times \Gamma_{t}, \\
V & =\left(1+\delta \mathrm{H}_{t}\right) u, & & \text { on } \bigcup_{t>0}\{t\} \times \Gamma_{t}, \\
\left.\Gamma_{t}\right|_{t=0} & =\Gamma_{0}, & & \text { at } t=0 .
\end{aligned}
$$

In the above equations $V$ denotes the front speed in normal (outward) direction $\nu_{t}$, and $\mathrm{H}_{t}$ its mean curvature. The function $g>0$ describes the concentration profile of a reservoir of the diffusing molecule whereas the positive constants $\varepsilon, \delta$ arise in the nondimensionalization process. They measure the deviation from a threshold concentration of the penetrant and the strength of the curvature term, respectively. Condition (1.3) models conservation of mass across the free boundary, whereas condition (1.4) is a phenomenological law which is capable of capturing the behavior observed in experiments know as case II or anomalous diffusion. The curvature term naturally appears if one assumes the front speed to be proportional to the local average concentration in a reference ball of radius $\delta>0$ rather than to its point-wise value. The system is clearly nonlinear and, even if the boundary conditions were linear, it still would be. Indeed, two different solutions have different domains of definitions and cannot be added. This typical feature of FBPs becomes apparent after the simple 
change of variables

$$
(\tau, \xi, \eta)=\left(t, x, \frac{y}{s(t, x)}\right), \hat{u}(\tau, \xi, \eta)=u(\tau, \xi, \eta s(\tau, \xi))
$$

which transforms the problem to a corresponding one on a fixed domain. To do so, it is assumed that the fixed boundary of the unknown domain is given by

$$
\Gamma_{0}=\mathbb{R}^{n-1} \times\{0\}
$$

and that the unknown moving boundary can be described as the graph of a function $s(t, \cdot)$. Latter assumption is motivated by (initial) condition (1.5). In the new variables, the domain becomes the strip

$$
S=\mathbb{R}^{n-1} \times[0,1]
$$

with the obvious boundaries denoted by $\Gamma_{j}, j=0,1$. Using the old notation for the new variables the system now reads

$$
\begin{array}{rlrl}
\varepsilon u_{t}-\triangle_{x} u-\frac{1+y^{2}|\nabla s|^{2}}{s^{2}} \partial_{y}^{2} u & =\varepsilon y \frac{\dot{s}}{s} \partial_{y} u-2 y \frac{1}{s}\left(\nabla s \mid \partial_{y} \nabla u\right) & & -y \frac{s \triangle s-2|\nabla s|^{2}}{s^{2}} \partial_{y} u \\
u & =g & & \text { on } \Gamma_{0} \\
-\frac{1+|\nabla s|^{2}}{s} \partial_{y} u & =(1+\varepsilon u) \dot{s}-(\nabla s \mid \nabla u) & & \text { on } \Gamma_{1} \\
\dot{s} & =\sqrt{1+|\nabla s|^{2}}\left(1+\delta \mathrm{H}_{t}\right) u & & \text { on } \Gamma_{1} \\
s(0, \cdot) & =0
\end{array}
$$

In [?] the quasi-stationary approximation $(\varepsilon=0)$ in two space dimensions was considered in the presence or absence of the mean curvature term in two space dimensions. Here there is no restriction on the spatial dimension and the full evolutionary problem is analyzed but the curvature effects are neglected $(\delta=0)$.

The main result of this paper is establishing the well-posedness of system (1.6)-(1.10). To do so, appropriate function spaces have to be used which are able to capture the regularity of the solution as well as its asymptotic behavior at the origin. The choice of function spaces is limited by the simultaneous presence of a singular parabolic problem and a Hamilton-Jacobi equation. Spaces of classical regularity are better suited for the latter and thus reduce the freedom of choice for the parabolic problem. A compromise can be reached by using spaces of classical Hölder regularity in space and of singular Hölder behavior in time. A number of preparatory results are needed; they are formulated in the next section. In section 3 elliptic and parabolic results for singular equations will be derived which play a crucial role in the existence proof given in the last section.

2. Preliminaries and Setting. In order to obtain existence results for system (1.6)-(1.10) a linearization procedure will be used in combination with maximal regularity results for the relevant linearized problems. The presence of the singularity complicates the analysis significantly since the relevant regularity results are not available and need to be derived first. The linearization procedure is meant to capture the leading order terms of the differential operators at the origin both with respect to their differentiation order and their singularity degree. 
Of interest in this paper are classical solutions. If such a solution existed, one would be able to guess from (1.7) and (1.9)-(1.10) that,

$$
s(t, x) \approx \operatorname{tg}(x) \text { for } t \approx 0
$$

This indicates that the relevant model problem which captures the leading order behavior of (1.6)-(1.8) has the form

$$
\begin{aligned}
\varepsilon u_{t}-\triangle_{x} u-\frac{1}{t^{2} c^{2}(x)} \partial_{y}^{2} u & =f(t, x, y) \text { in }(0, \infty) \times S, \\
u & =g(t, x) \quad \text { on }(0, \infty) \times \Gamma_{0}, \\
\frac{1}{t} \partial_{y} u & =h(t, x) \quad \text { on }(0, \infty) \times \Gamma_{1},
\end{aligned}
$$

where in the situation at hand $g$ is independent of the time variable and $c=g$. The more general case of $c$ independent of $g$ is, however, of interest and motivates the different notation. For reasons which can be guessed and will become apparent later, both parabolic $(\varepsilon=1)$ and elliptic $(\varepsilon=0)$ regularity results are needed in the analysis of (2.1)-(2.3). The main ingredients needed to derive such regularity results are vector-valued Fourier multiplier theorems and the use of spaces of singularly Hölder continuous functions in the time variable and the construction of an evolution operator for singular families of generators. The first are crucial in the analysis of the elliptic problem with time frozen and the latter allow for the quantitative characterization of the singular behavior in the origin $(t=0)$ both in the elliptic and the parabolic case (where maximal regularity results are needed).

For the sake of completeness the formulation of the relevant Fourier multiplier theorem and the definition of the classes of singular Hölder continuous functions needed in the analysis are given here. The basic observation illuminating the reason for their combined use will also be presented in this section.

Assume that $E$ is a given Banach space and that $T>0$ and $\beta \in(0,1)[\cup\{1-\}]$, then the standard Hölder [Lipschitz] space is given by

$$
\mathrm{C}^{\beta}([0, T], E):=\left\{f \in \mathrm{C}([0, T], E) \mid[u]_{\beta,[0 T]}:=\sup _{t \neq s} \frac{|u(t)-u(s)|}{|t-s|^{\beta}}<\infty\right\}
$$

with norm

$$
\|\cdot\|_{\beta}=\|\cdot\|_{\infty,[0, T]}+[\cdot]_{\beta,[0, T]} \cdot
$$

If $\beta=1$ is chosen, one obtains the space of Lipschitz continuous functions. To distinguish it from the space of continuously differentiable functions the notational device $\beta=1-$ is used. This means that $\beta=1$ for all practical purposes except in the notation for the space which becomes $\mathrm{C}^{1-}$. Singular counterparts are given by

$$
\mathrm{C}_{\beta}^{\beta}((0, T], E):=\left\{f \in \mathrm{B}((0, T], E) \mid\left[t \mapsto t^{\beta} u(t)\right] \in \mathrm{C}^{\beta}((0, T], E)\right\}
$$

with weighted norm defined through

$$
\|\cdot\|_{\beta, \beta}:=\|u\|_{\infty,(0, T]}+\left[(\cdot)^{\beta} u\right]_{\beta,(0, T]} .
$$

The symbol denoting the time interval in the notation for the norm will be dropped in the sequel with the understanding that the interval of definition does not contain 
the origin for singular spaces, whereas it does for regular ones. The following closed subspace of regular Hölder functions will also be useful

$$
\mathrm{C}_{0}^{\beta}([0, T], E):=\left\{f \in \mathrm{C}^{\beta}([0, T], E) \mid f(0)=0\right\} .
$$

The vector-valued Fourier multiplier theorem which is needed here can be found in [?, Theorem 6.2]. We reproduce it here for the ease of the reader. The following symbol class

$$
\begin{aligned}
& \mathcal{S}^{m}\left(E_{0}, E_{1}\right):=\left\{a \in \mathrm{C}^{n+1}\left(\mathbb{R}^{n} \backslash\{0\}, \mathcal{L}\left(E_{0}, E_{1}\right)\right) \mid\right. \\
&\left.\sup _{\xi \in \mathbb{R}^{n}}\left|\left[1+|\xi|^{2}\right]^{(m+|\alpha|) / 2} \partial^{\alpha} a(\xi)\right|_{\mathcal{L}\left(E_{0}, E_{1}\right)}<\infty,|\alpha| \leq n+1\right\}
\end{aligned}
$$

where $E_{0}$ and $E_{1}$ are Banach spaces and $m \in \mathbb{Z}$, is instrumental in the formulation of the result. Given a symbol $a \in \mathcal{S}^{m}\left(E_{0}, E_{1}\right)$, an operator can be associated to it by

$$
a(D):=\mathcal{F}^{-1} a \mathcal{F}
$$

through conjugation with the vector-valued Fourier transform

$$
\mathcal{F} \in \mathcal{L}_{i s}\left(\mathcal{S}\left(\mathbb{R}^{n}, E_{j}\right)\right) \cap \mathcal{L}_{i s}\left(\mathcal{S}^{\prime}\left(\mathbb{R}^{n}, E_{j}\right)\right), j=0,1,
$$

where $\mathcal{S}$ and $\mathcal{S}^{\prime}$ are the operator-valued Schwartz' spaces of fast decaying test functions and of tempered distributions, respectively. They are endowed with their natural topology.

Theorem 2.1. [?, Theorem 6.2] Suppose that $\mathcal{B} \in\{B, \stackrel{\circ}{B}, b\}$ and that $m \in \mathbb{R}$. Then

$$
[a \mapsto a(D)] \in \mathcal{L}\left(\mathrm{S}^{m}\left(E_{0}, E_{1}\right), \mathcal{L}\left(\mathcal{B}_{p, q}^{s}\left(\mathbb{R}^{n}, E_{0}\right), \mathcal{B}_{p, q}^{s+m}\left(\mathbb{R}^{n}, E_{1}\right)\right)\right)
$$

for $s \in \mathbb{R}$ and $p, q \in[1, \infty]$.

The symbol $B$ denotes the regular Besov spaces whereas $\stackrel{\circ}{B}_{p, q}^{s}$ and $b_{p, q}^{s}$ denote the closures of $\mathcal{S}$ and $B_{p, q}^{s+1}$ in $B_{p, q}^{s}$, respectively. Various equivalent definitions are given in [?]. Here only regular Besov spaces with $p=q=\infty$ of positive fractional order $s \in \mathbb{R}^{+} \backslash \mathbb{N}$ are used in which case one has

$$
B_{\infty, \infty}^{s}=\operatorname{BUC}^{s}\left(\mathbb{R}^{n}, E\right),
$$

where the space on the right is the standard space of bounded and uniformly Hölder continuous functions given by

$$
\operatorname{BUC}^{s}\left(\mathbb{R}^{n}, E\right):=\left\{f \in \mathrm{BUC}^{[s]}\left(\mathbb{R}^{n}, E\right)\left|\partial^{\alpha} f \in \mathrm{BUC}^{s-[s]}\left(\mathbb{R}^{n}, E\right) \forall\right| \alpha \mid \leq[s]\right\} .
$$

A crucial observation connecting the dilation of symbols of type (2.5) and singular Hölder spaces was already obtained in [?]. It allows one to deal with singular elliptic and, eventually, singular parabolic boundary value problems.

Proposition 2.2. [?, Lemma 2.5] Assume that $a \in \mathrm{S}^{m}\left(E_{0}, E_{1}\right)$ for some $m \in \mathbb{Z}$. Then

$$
\left[t \mapsto t^{m} \sigma_{t} a\right] \in \mathrm{C}_{1}^{1-}\left((0, T], \mathrm{S}^{m}\left(E_{0}, E_{1}\right)\right)
$$


for $\sigma_{t}(a):=a(t \cdot)$.

Remark 2.1. A straightforward adaptation of the proof given in [?] of Proposition 2.2 also shows that

$$
\left[t \mapsto t^{m} \sigma_{t} a\right] \in \mathrm{C}^{1-}\left([0, T], \mathrm{S}^{m-1}\left(E_{0}, E_{1}\right)\right)
$$

The importance of this proposition lies in the fact that multiplication of singularly Hölder continuous functions is well-defined and continuous as a map defined in various combinations of spaces

$$
\begin{aligned}
& \mathrm{C}_{\alpha}^{\alpha} \times \mathrm{C}_{\beta}^{\beta} \rightarrow \mathrm{C}_{\beta}^{\beta} \\
& \mathrm{C}_{\alpha}^{\alpha} \times \mathrm{C}_{0}^{\beta} \rightarrow \mathrm{C}_{0}^{\beta} \\
& \mathrm{C}_{0}^{\alpha} \times \mathrm{C}_{0}^{\beta} \rightarrow \mathrm{C}_{-\alpha}^{\beta} \\
& \mathrm{C}_{0}^{\alpha} \times \mathrm{C}_{\beta}^{\beta} \rightarrow \mathrm{C}_{\beta-\alpha}^{\beta}
\end{aligned}
$$

for $\alpha, \beta \in(0,1) \cup\{1-\}$ and $\beta \leq \alpha$. The proof is elementary and can be found in [?] along with the (natural) definition of the spaces for negative lower indices.

Finally optimal regularity results are yet another essential ingredient to deal with the full nonlinear problem. It provides maximal regularity results for the relevant class of singular parabolic problems in classes of singularly Hölder continuous functions which are, as they need to be, perfectly compatible with the corresponding results for singular elliptic problems in the same class of functions. The result needed here has been derived in [?] and is formulated in the next theorem. It gives conditions for the well-posedness of the singular abstract Cauchy problem

$$
\dot{u}-A(t) u=f(t), t>0
$$

for a Banach space valued function $u:(0, T] \rightarrow E_{0}$ and a singular family of "elliptic operators" $A$ (that is, of generators of analytic semi-groups). The symbol $\mathcal{H}^{-}\left(E_{0}, \omega\right)$ denotes the class of generators of exponentially decaying semi-groups which are not necessarily strongly continuous (as introduced by [?]).

Theorem 2.3. [?, Corollary 3.3] Assume that A satisfies the following assumptions

$$
\begin{aligned}
& \text { (i) } A(t) \in \mathcal{H}^{-}\left(E_{0}, \omega\right), t>0 \\
& \text { (ii) }\left\|[A(t)-A(s)] A^{-1}(\tau)\right\|_{\mathcal{L}\left(E_{0}\right)} \leq c \frac{t-s}{t} \text { and } \\
& \left\|[A(t)-A(s)](-A)^{-\rho}(\tau)\right\|_{\mathcal{L}\left(E_{0}\right)} \leq c(t-s), \\
& \text { (iii) } \lim _{t \rightarrow 0} A^{-1}(t)=0
\end{aligned}
$$

for some $\rho \in(1,2)$ and $0<\tau \leq s \leq t \leq T$. Let $f \in \mathrm{C}_{\gamma}^{\beta}\left((0, T], E_{0}\right)$ for some $\beta \in(0,1)$ and $\gamma=0, \beta$. Then (2.10) has a unique solution $u \in \mathrm{C}_{\gamma}^{\beta}\left((0, T], E_{0}\right)$ satisfying

$$
\dot{u}, A u \in \mathrm{C}_{\gamma}^{\beta} \text { and }\|\dot{u}\|_{\beta, \gamma}+\|A u\|_{\beta, \gamma} \leq c\|f\|_{\beta, \gamma}
$$

where $\|\cdot\|_{\beta, 0}=\|\cdot\|_{\beta}$.

Using a combination of elliptic and parabolic estimates it will be possible to obtain a satisfactory regularity theory for (2.1)-(2.3). The latter will used in the analysis of the full evolution system (1.6)-(1.10) for $\delta=0$. 
3. Elliptic and Parabolic Estimates. In order to use the results presented in the previous section in the analysis of (1.6)-1.10), appropriate function spaces have to be chosen in which to work. The choice can typically be justified by balancing the regularities in the nonlinear equations in such a way that a fixed point argument can be applied to the set of equations. Whereas a variety of function spaces of Sobolev type are available for dealing with elliptic and parabolic problems, the fact that the system of interest contains a Hamilton-Jacobi equation for the evolution of $s$, which, in turn, appears in differentiated form as a coefficient in the equations for the evolution of $u$ restricts the choice to spaces of classical regularity for $s$. It is indeed impossible to work in Sobolev-Slobodeckii spaces for $u$ as the loss of regularity incurred in taking traces on the boundary cannot be made up for by (1.9) which does not possess any regularizing effect. It becomes clear that spaces of classical regularity for both $u$ and $s$ need to be chosen. In order to use the Fourier multiplier Theorem 2.1] the choice is therefore reduced to spaces of bounded uniformly continuous functions in the $x$ variable. As far as the $y$ variable is concerned a good choice is given by the space of continuous functions as will become clear later. The "base" space $E_{0}$ for $u$ is therefore chosen as

$$
E_{0}:=\mathrm{BUC}^{1+\alpha}\left(\mathbb{R}^{n}, \mathrm{C}(0,1)\right)
$$

for $\alpha \in(0,1)$ and where $\mathrm{C}(0,1)$ denotes the standard space of continuous functions on $[0,1]$. The space with one less regularity degree in $x$ would seem like a more natural choice but the choice made here makes it much easier to deal with inhomogeneous Neumann boundary conditions such as (1.8). This point will become clear later in the analysis in Section [3.3 In view of the singular non-autonomous nature of the problem, the standard procedures ([?]) to deal with inhomogeneous (nonlinear) boundary conditions cannot be utilized.

It follows that a proper choice of "base" space for the full evolutionary problem is given by

$$
\mathbb{E}_{0}:=\mathrm{C}_{\beta}^{\beta}\left((0, T], \mathrm{BUC}^{1+\alpha}\left(\mathbb{R}^{n}, \mathrm{C}(0,1)\right)\right)
$$

for $u$ and by

$$
\mathbb{S}:=\mathrm{C}^{1+\beta}\left([0, T], \mathrm{BUC}^{2+\alpha}\left(\mathbb{R}^{n}\right)\right) \cap \mathrm{C}^{\beta}\left([0, T], \mathrm{BUC}^{3+\alpha}\left(\mathbb{R}^{n}\right)\right)
$$

for $s$. A closer look at the underlying elliptic problem $(\varepsilon=0)$ is necessary in order to better deal with the inhomogeneous boundary terms in (2.1)-(2.3). The operator $-\triangle_{x}-\frac{1}{t^{2} g^{2}(x)} \partial_{y}^{2}$ has clearly non-constant coefficients and multiplier Theorem 2.1 is formulated only in the translation invariant case. It therefore needs to be shown that localization arguments apply in the operator-valued setting considered here.

The first step is, however, to obtain regularity results in the constant coefficient case.

3.1. The Elliptic Case - Constant Coefficients. Consider problem (2.1)(2.3) and assume that $\varepsilon=0$ and that $c$ is a constant which can be assumed to be 1 without loss of generality. Then taking a Fourier transform in the $x$-variables, one obtains a parameter dependent boundary value problem for $\hat{u}$ which can be solved explicitly. The solution has the structure

$$
\hat{u}(t, \xi, \cdot)=t^{2} \sigma_{t} c(\xi, \cdot) \hat{f}+t \sigma_{t} b(\xi, \cdot) \hat{h}+\sigma_{t} a(\xi, \cdot) \hat{g}
$$


where $\sigma_{t}$ denotes dilation by $t>0$ and the operator-valued symbols $a, b, c$ are given by

$$
a(\xi, \cdot)=\frac{\cosh (|\xi|(1-\cdot))}{\cosh (|\xi|)}, b(\xi, \cdot)=\frac{\sinh (|\xi| \cdot)}{|\xi| \cosh (|\xi|)}, c(\xi, \cdot)=\left(|\xi|^{2}+C\right)^{-1} .
$$

The first two symbols have to be considered as multiplication operator valued with respect to the variable $y$, whereas the last contains the sectorial operator $C$ which is the operator $-\partial_{y}^{2}$ on $\mathrm{C}(0,1)$ with domain

$$
\operatorname{dom}(C)=\left\{u \in \mathrm{C}^{2}(0,1) \mid u(0)=0, \partial_{y} u(1)=0\right\}=: \mathrm{C}_{0,0}^{2}(0,1) .
$$

The spaces

$$
\mathrm{C}_{0}^{k}:=\left\{u \in \mathrm{C}^{k}(0,1) \mid u(0)=0\right\}, k=0,1
$$

will also be needed. Proposition 2.2 can be used in combination with the multiplications (2.6)-(2.9) to obtain regularity results in weighted Hölder spaces provided (3.5) are in the appropriate symbol class.

Theorem 3.1. The symbols (3.5) satisfy

$$
\begin{aligned}
& a \in \mathcal{S}^{0}(\mathrm{C}(0,1), \mathrm{C}(0,1)) \\
& b \in \mathcal{S}^{k}\left(\mathrm{C}(0,1), \mathrm{C}_{0}^{1-k}(0,1)\right), \\
& \quad c \in \mathcal{S}^{j}\left(\mathrm{C}(0,1), \mathrm{C}_{0,0}^{2-j}(0,1)\right)
\end{aligned}
$$

for $k=0,1$ and $j=0,1,2$. The notations for the spaces have to be interpreted so that boundary conditions are imposed only if enough regularity is available but otherwise disregarded.

Proof. Take the last symbol first. It is known that the operator $C$ is sectorial and invertible (cf. [?]). It follows that

$$
\left\|\left(|\xi|^{2}+C\right)^{-1}\right\|_{\mathcal{L}\left(\mathrm{C}, \mathrm{C}^{2-j}\right)} \leq c \frac{1}{1+|\xi|^{j}}, j=0,1,2
$$

As for the derivatives, it is seen by induction that, for $\xi \neq 0$,

$$
\partial^{\alpha}\left(|\xi|^{2}+C\right)^{-1}=\left(|\xi|^{2}+C\right)^{-1} \sum_{|\beta| \leq|\alpha|} p_{\alpha, \beta}(\xi)\left(|\xi|^{2}+C\right)^{-|\beta|}
$$

for polynomials $p_{\alpha, \beta}$ of degree at most $|\beta|$. The estimate therefore follows from (3.7). Next consider the "boundary symbols" $a$ and $b$. A simple series expansion reveals that both symbols are actually analytic functions of $\xi^{2}$ (and $y$ of course). It follows that they are smooth for all $\xi \in \mathbb{R}^{n}$ in spite of appearances. It is therefore legitimate to concentrate on the decay properties of the symbols without necessarily using the nowhere vanishing weight $\left(1+|\xi|^{2}\right)^{1 / 2}$ needed in the definition of the symbol class. Observe that both are smooth functions of $y$ regardless of $\xi$. Consequently they define multiplication operators for $\mathrm{C}^{2-k}(0,1)$ and $k=0,1,2$. Also observe that, for $b$, the smoothing effect in $x$ is due to the decay in $\xi$. They clearly satisfy the required boundary conditions. They further satisfy the relations

$$
\begin{gathered}
\partial_{y} b(\xi, y)=a(\xi, 1-y), \partial_{y}^{2} b(\xi, y)=|\xi|^{2} b(\xi, y), \\
\partial_{y} a(\xi, y)=-|\xi|^{2} b(\xi, 1-y), \partial_{y}^{2} a(\xi, y)=|\xi|^{2} a(\xi, y),
\end{gathered}
$$


which make it easier to derive their mapping and symbol properties from one another. For instance, if it were known that $a, b \in \mathcal{S}^{0}(\mathrm{C}, \mathrm{C})$ the first relation would imply that $\partial_{y} b \in \mathcal{S}^{0}(\mathrm{C}, \mathrm{C})$ and therefore that $b \in \mathcal{S}^{0}\left(\mathrm{C}, \mathrm{C}^{1}\right)$. The last one, on the same assumptions, would show that $a \in \mathcal{S}^{-2}\left(\mathrm{C}, \mathrm{C}^{2}\right)$. More details are now given for the analysis of symbol $a$. Corresponding claims for the symbol $b$ can be obtained by similar calculations. The claim is that $a \in \mathcal{S}^{0}(C, C)$. It is plain that

$$
\sup _{\xi \in \mathbb{R}^{n}} \sup _{y \in[0,1]}|a(\xi, y)|<\infty .
$$

As for the first derivatives in $\xi$ one has

$$
\begin{gathered}
\partial_{j} a(\xi, y)=\frac{\xi_{j}}{|\xi|} \frac{1}{\cosh (|\xi|)}[(1-y) \sinh (|\xi|(1-y))-\tanh (|\xi|) \cosh (|\xi|(1-y))]= \\
\frac{\xi_{j}}{|\xi|} a(\xi, y) \underbrace{[(1-y) \tanh (|\xi|(1-y))-\tanh (|\xi|)]}_{=: d(\xi, y)} .
\end{gathered}
$$

The maximum in $y$ is either taken on the boundary $y=0,1$ or in the interior. On the boundary it either vanishes or is exponentially decaying in $|\xi|$. It therefore only needs to be controlled in the interior to conclude its estimation. Only the term in brackets in the right-hand-side in the first line of (3.8) depends on $y$. Setting its first $y$-derivative equal to zero, one arrives at the equation

$$
\frac{\tanh (|\xi|(1-y))}{(1-y)|\xi|}=\frac{1}{|\xi| \tanh (|\xi|)-1}
$$

which, for $|\xi|$ large, is asymptotic to

$$
\frac{1}{(1-y)|\xi|} \sim \frac{1}{|\xi|}
$$

Thus the maximum is located at $y \sim \frac{1}{|\xi|}$ for large $|\xi|$. As for the maximal value for large $|\xi|$ one has

$$
\partial_{j} a\left(\xi, \frac{1}{|\xi|}\right) \sim \frac{\xi_{j}}{|\xi|} a\left(\xi, \frac{1}{|\xi|}\right) \frac{1}{|\xi|}
$$

which entails the desired estimate

$$
\sup _{x \in \mathbb{R}^{n}} \sup _{y \in[0,1]}\left(1+|\xi|^{2}\right)^{1 / 2}\left|\partial_{j} a(\xi, y)\right|<\infty .
$$

Equation (3.9) follows from

$$
\left(1-\frac{1}{|\xi|}\right) \tanh (|\xi|-1)-\tanh (|\xi|)
$$

observing that $\tanh (s)=1$ up to exponentially small terms in $s$ for $s>0$ large, that is,

$$
|\tanh (s)-1| \leq c e^{-3 s} s>0 .
$$


The second derivative satisfies

$$
\partial_{i} \partial_{j} a(\xi, y)=\frac{\xi_{i} \xi_{j}}{|\xi|^{3}} a(\xi, y) d(\xi, y)+\frac{\xi_{j} \xi_{j}}{|\xi|^{2}} a(\xi, y) d^{2}(\xi, y)+\frac{\xi_{j}}{|\xi|} a(\xi, y) \partial_{j} d(\xi, y)
$$

The first term in the right-hand-side can be seen to decay like $1 /|\xi|^{2}$ in view of the explicit pre-factor and the estimate obtained for the first derivative (3.8). The second and third terms add up to

$$
\frac{\xi_{i} \xi_{j}}{|\xi|^{2}} a(\xi, y)\left[(1-y)^{2}-1-2(1-y) \tanh (|\xi|) \tanh (|\xi|(1-y))+2 \tanh ^{2}(|\xi|)\right]
$$

Similar calculations as those performed for the first derivative show that the maximum is now attained at

$$
y \sim \frac{2}{|\xi|}
$$

and it amounts to

$$
\partial_{i} \partial_{j} a\left(\xi, \frac{2}{|\xi|}\right)-\frac{\xi_{i} \xi_{j}}{|\xi|^{3}} a\left(\xi, \frac{2}{|\xi|}\right) d\left(\xi, \frac{2}{|\xi|}\right) \sim \frac{4}{|\xi|^{2}} .
$$

Latter follows from

$$
\left(1-\frac{2}{|\xi|}\right)^{2}-1-2\left(1-\frac{2}{|\xi|}\right) \tanh (|\xi|-2) \tanh (|\xi|)+2 \tanh ^{2}(|\xi|) \sim \frac{4}{|\xi|^{2}}
$$

which uses (3.10) again. In conclusion it is obtained that

$$
\sup _{\xi \in \mathbb{R}^{n}} \sup _{y \in[0,1]}\left(1+|\xi|^{2}\right)\left|\partial_{i} \partial_{j} a(\xi, y)\right|<\infty .
$$

Comparing with the proof of [?, Lemma 2.2], it is observed that the arguments are almost identical. The only difference are the additional factors like $\frac{\xi_{j}}{|\xi|}$ for the first derivative or $\frac{\xi_{i} \xi_{j}}{|\xi|^{3}}$ and $\frac{\xi_{i} \xi_{j}}{|\xi|^{2}}$ for the second. Terms containing these can always be handled through their explicit dependence on $\xi$ and the estimates from previous derivatives. The inductive argument used in [?] can therefore be adapted to the current situation and leads to the desired result. $\square$

Combining these results with Theorem 2.1] Proposition 2.2 and (2.6)-(2.9) the next important theorem is obtained. To simplify its formulation we introduce the abbreviated notation

$$
\mathrm{C}_{?}^{?} \mathrm{BUC}^{?} \mathrm{C}_{?, ?}^{?}
$$

for the function spaces

$$
\mathrm{C}_{?}^{?}\left((0, T], \mathrm{BUC}^{?}\left(\mathbb{R}^{n}, \mathrm{C}_{?, ?}^{?}(0,1)\right)\right)
$$

where the question marks can be substituted by any of the relevant regularity and singularity parameters.

Theorem 3.2. Let $\alpha, \beta \in(0,1)$ and $\gamma=0, \beta$. Given

$$
(f, g, h) \in \mathrm{C}_{\gamma}^{\beta} \mathrm{BUC}^{1+\alpha} \mathrm{C} \times \mathrm{C}_{\gamma}^{\beta} \mathrm{BUC}^{3+\alpha} \times \mathrm{C}_{\gamma}^{\beta} \mathrm{BUC}^{2+\alpha}
$$


there exists a unique solution $u$ of (2.1)-(2.3) for $c \equiv$ const which belongs to the space

$$
\mathbb{E}_{1}:=\left\{u \in \mathbb{E}_{0} \mid \partial_{x}^{\alpha}\left(\frac{\partial_{y}}{t}\right)^{j} u \in \mathbb{E}_{0} \text { for }|\alpha|, j=0,1,2 \text { s.t. }|\alpha|+j \leq 2\right\}
$$

where

$$
\mathbb{E}_{0}:=\mathrm{C}_{\gamma}^{\beta} \mathrm{BUC}^{1+\alpha} \mathrm{C}
$$

It is given by

$$
u=\underbrace{\mathcal{F}^{-1} t^{2} \sigma_{t} c \mathcal{F}}_{=: A(t)^{-1}} f+\underbrace{\mathcal{F}^{-1} t \sigma_{t} b \mathcal{F}}_{=: R_{N}(t)} h+\underbrace{\mathcal{F}^{-1} \sigma_{t} a \mathcal{F}}_{=: R_{D}(t)} g .
$$

Modulo the singularity, the terms in the above representation clearly point to their asymptotic behavior at the origin. This result needs to be extended to the nonconstant coefficient case. This is done in the next section.

REMARK 3.1. To make it easier to refer back to the above result in the case $\gamma=0$, the corresponding spaces will be denoted by $\mathbb{E}_{k, 0}, k=0,1$.

3.2. The Elliptic Non-constant Coefficient Case. In this section we adapt an abstract formulation of a localization argument proposed by Angenent in [?] (see also [?, ?]) for $\mathbb{R}^{n}$ to cover the case of boundary value problems in an operator-valued context. It is used in combination with the Fourier multiplier Theorem 2.1 to give the basic regularity results needed for the singular elliptic boundary value problem underlying (2.1)-(2.3) in the case of constant coefficients. It should be observed that localization arguments rely on perturbation results for differential operators by lower order terms. These are small compared to the leading order operator only in a qualitative sense. Perturbation results can therefore only be applied for the resolvents with large $\lambda$ where the decay properties of the inverse to the leading order operator yields smallness of the lower order terms. The structure of the singular operators considered here can be exploited in order to avoid shifting the leading order operator and in order to obtain direct invertibility results. The standard argument of course shows that non-constant coefficient operators are sectorial whenever their constant coefficient counterpart is (see [?]). This remains valid here and can be used if the time variable is kept fixed.

The goal of this section is to extend the validity of Theorem 3.2 to the nonconstant coefficient singular Boundary Value Problem

$$
\left(\mathcal{A}(t), \gamma_{0}, \frac{1}{t} \gamma_{1} \partial_{y}\right): \mathbb{E}_{1} \rightarrow \mathbb{E}_{0} \times \partial \mathbb{E}_{1}
$$

where $\mathcal{A}(t)$ is the elliptic operator driving the evolution in 2.1) and the "boundary space" $\partial \mathbb{E}_{1}$ is defined by

$$
\partial \mathbb{E}_{1}=\mathrm{C}_{\gamma}^{\beta} \mathrm{BUC}^{3+\alpha} \times \mathrm{C}_{\gamma}^{\beta} \mathrm{BUC}^{2+\alpha}=: \partial_{D} \mathbb{E}_{1} \times \partial_{N} \mathbb{E}_{1}, \gamma=0, \beta .
$$

Observe that Theorem 3.2 simply states that the above singular Boundary Value Problem has a bounded inverse in the given topologies and for constant coefficients. The localization procedure proposed in [?] is abstract and is based on the concept of resolution. A resolution of a Banach space $E$ is simply a triple $(F, \varepsilon, \delta)$ where $F$ is a Banach space and the maps $\delta: E \rightarrow F$ and $\varepsilon: F \rightarrow E$ satisfy $\varepsilon \circ \delta=\operatorname{id}_{E}$. The operator $\delta$ plays the role of the localizing operator, whereas $\varepsilon$ resynthesizes the local 
contributions. If the space $E$ has subspaces of interest, they should correspond to "similar subspaces" of $F$ and should be left invariant by the resolution maps. The localization operator can be constructed as follows

$$
\delta: \mathbb{E}_{j} \rightarrow \oplus_{k \in \mathbb{Z}} \mathbb{F}_{j}^{k}, u \mapsto\left(u_{k}\right)_{k \in \mathbb{Z}^{n}}:=\left(u \varphi_{k, r}\right)_{k \in \mathbb{Z}^{n}}, j=0,1
$$

where $\mathbb{F}_{j}^{k}=\mathbb{E}_{j}$ for $k \in \mathbb{Z}^{n}$ and the sequence space $\oplus_{k \in \mathbb{Z}} \mathbb{F}_{j}^{k}$ is endowed with the supremum norm $\sup _{k \in \mathbb{Z}}\left\|u_{k}\right\|_{\mathbb{F}_{j}}$. The maps $\left(\varphi_{k, r}^{2}\right)_{k \in \mathbb{Z}^{n}}$ are chosen as to form a smooth resolution of the identity in $\mathbb{R}^{n}$

$$
\sum_{k \in \mathbb{Z}^{n}} \varphi_{k, r}^{2} \equiv 1
$$

subordinated to a cover by cubes $Q_{k \in \mathbb{Z}^{n}, r}$ of fixed side length $r>0$ obtained by translation and dilation of the standard cube of side size $r=1$ centered at the origin. The support of $\varphi_{k, r}$ should contain $Q_{k, r}$ and be contained in the union of at most finitely many adjacent cubes. The synthesis operator is then given by

$$
\varepsilon: \oplus_{k \in \mathbb{Z}} \mathbb{F}_{j}^{k} \rightarrow \mathbb{E}_{j},\left(u_{k}\right)_{k \in \mathbb{Z}^{n}} \mapsto \sum_{k \in \mathbb{Z}^{n}} u_{k} \varphi_{k, r}
$$

in which case the relation defining a resolution is clearly satisfied. Observe that the mappings $\delta$ and $\varepsilon$ are continuous. The idea is to define an operator on $\oplus_{k \in \mathbb{Z}} \mathbb{F}_{1}$ which parallels the Boundary Value Problem (3.11) and to use its inverse to approximate the desired inverse of (3.11) via the use of the maps $\varepsilon$ and $\delta$. Define the operator $\left(\mathcal{A}^{\prime}, \mathcal{B}^{\prime}\right)$ on $\oplus_{k \in \mathbb{Z}} \mathbb{F}_{1}$ through

$$
\left(\mathcal{A}^{k}, \mathcal{B}^{k}\right) u_{k}=\left(-\triangle_{x} u_{k}-\frac{1}{t^{2} c_{k}(x)} \partial_{y}^{2} u_{k}, \gamma_{0} u_{k}, \frac{1}{t} \gamma_{1} \partial_{y} u_{k}\right)
$$

where $\left.c_{k}\right|_{\operatorname{supp}\left(\varphi_{k, r}\right)}=\left.c\right|_{\operatorname{supp}\left(\varphi_{k, r}\right)}$ and $c_{k}$ is otherwise smoothly extended without increasing its norm to the whole space (the details of the extension procedure can be found in [?, ?]). The Boundary Value Problem $\left(\mathcal{A}^{k}, \mathcal{B}^{k}\right)$ can be made arbitrarily close to a constant coefficient one, say by substituting $c_{k}$ by $c_{k}\left(x_{k}\right)$ and making $r$ small. It is therefore invertible and enjoys the properties claimed in Theorem 3.2 The diagonal operator $\left(\mathcal{A}^{\prime}, \mathcal{B}^{\prime}\right)$ is then invertible itself and maps $\oplus_{k \in \mathbb{Z}}\left[\mathbb{F}_{1} \times \partial \mathbb{F}_{1}\right]$ to $\oplus_{k \in \mathbb{Z}} \mathbb{F}_{0}$ where $\partial \mathbb{F}_{1}$ is defined in the obvious way. Denote by $\left[\begin{array}{lll}A^{\prime}(t)^{-1} & R_{D}^{\prime}(t) & R_{N}^{\prime}(t)\end{array}\right]$ its inverse. Then

$$
\varepsilon\left[A^{\prime}(t)^{-1} \quad R_{D}^{\prime}(t) \quad R_{N}^{\prime}(t)\right] \delta
$$

should represent an approximation to the solution operator for the singular boundary value problem (2.1)-(2.3). In order to show this, it needs to be shown that (3.13) is an approximate left and right inverse. Starting with the latter, compute

$$
\begin{aligned}
& {\left[\begin{array}{c}
\mathcal{A}(t) \\
\gamma_{0} \\
\frac{1}{t} \gamma_{1} \partial_{y}
\end{array}\right] \varepsilon\left[\begin{array}{lll}
A^{\prime}(t)^{-1} & R_{D}^{\prime}(t) & \left.R_{N}^{\prime}(t)\right] \delta \\
\end{array}\right.} \\
& =\left[\begin{array}{ccc}
\operatorname{id}_{\mathbb{E}_{0}}+[\mathcal{A}(t), \varepsilon] A^{\prime}(t)^{-1} \delta & {[\mathcal{A}(t), \varepsilon] R_{D}^{\prime}(t) \delta} & {[\mathcal{A}(t), \varepsilon] R_{N}^{\prime}(t) \delta} \\
0 & \operatorname{id}_{\gamma_{0} \mathbb{E}_{1}} & 0 \\
0 & 0 & \operatorname{id}_{\gamma_{1} \partial_{y} \mathbb{E}_{1}}
\end{array}\right]
\end{aligned}
$$


where $[\mathcal{A}(t), \varepsilon]=\mathcal{A}(t) \varepsilon-\varepsilon \mathcal{A}(t)^{\prime}$. Invertibility would follow if

$$
\operatorname{id}_{\mathbb{E}_{0}}+[\mathcal{A}(t), \varepsilon] A^{\prime}(t)^{-1} \delta
$$

were invertible. Here the specific structure of the operator $\mathcal{A}(t)$ needs to be exploited since standard perturbation arguments would only allow to show that the resolvent $\mathcal{R}(\lambda, \mathcal{A})$ exists for large enough $\lambda$, in which case the lower order commutator term is small. The commutator term (3.15) can be computed to give

$$
\sum_{k \in \mathbb{Z}^{n}}\left[2\left(\nabla \varphi_{k, r} \mid \nabla \cdot\right)+\triangle \varphi_{k, r} \cdot\right]\left[-\triangle_{x}-\frac{1}{t^{2} c_{k}^{2}(x)} \partial_{y}^{2}\right]^{-1} \varphi_{k} f .
$$

To see that the inverse appearing in (3.16) does indeed exist on the desired spaces, observe that $r>0$ can be chosen such that

$$
-\triangle_{x}-\frac{1}{t^{2} c_{k}^{2}(x)} \partial_{y}^{2}+\triangle_{x}+\frac{1}{t^{2} c_{k}^{2}\left(x_{k}\right)} \partial_{y}^{2}=\left[\frac{1}{c_{k}^{2}(x)}-\frac{1}{c_{k}^{2}\left(x_{k}\right)}\right] \frac{1}{t^{2}} \partial_{y}^{2}
$$

is small in $\mathcal{L}\left(\mathbb{E}_{1}, \mathbb{E}_{0}\right)$. The realization $A^{k}$ of $\mathcal{A}^{k}$ with homogeneous boundary conditions is therefore invertible and the constant coefficient estimates of Theorem (3.2) carry over to it (with larger constants, of course). Now, the estimates

$$
\begin{aligned}
\left\|2 \nabla \varphi_{k, r} \nabla\left[-\triangle_{x}-\frac{1}{t^{2} c_{k}^{2}(x)} \partial_{y}^{2}\right]^{-1}\right\|_{\mathcal{L}\left(\mathbb{E}_{0}\right)} & \leq c T \text { and } \\
& \left\|\triangle \varphi_{k, r}\left[-\triangle_{x}-\frac{1}{t^{2} c_{k}^{2}(x)} \partial_{y}^{2}\right]^{-1}\right\|_{\mathcal{L}\left(\mathbb{E}_{0}\right)} \leq c T^{2}
\end{aligned}
$$

show that the commutator is indeed small, at least for $T$ small enough. Here the decay properties of the resolvent as $t \rightarrow 0$ are used as a substitute for making $\lambda$ large in the standard argument. Heuristically this makes sense, since making $t$ small, just as making $\lambda$ large, makes the operator "more and more elliptic". This is due to the specific nature of the singular operator. Since the difficulty of the problem stems from the origin, making $T$ small does not in any way weaken the result.

Next it needs to be shown that (3.13) is also a good approximation for a left inverse. To that end, observe that

$$
u-\varepsilon\left(\mathcal{A}^{\prime}(t), \mathcal{B}^{\prime}(t)\right)^{-1} \delta(\mathcal{A}(t), \mathcal{B}(t)) u
$$

is the same as

$$
u-\left(\varepsilon A^{\prime}(t)^{-1} \delta \mathcal{A}(t) u+\varepsilon R_{D}^{\prime}(t) \delta \gamma_{0} u+\varepsilon R_{N}^{\prime}(t) \delta \gamma_{1} \frac{\partial_{y}}{t} u\right)=\varepsilon A^{\prime}(t)^{-1}[\mathcal{A}(t), \delta] u
$$

because

$$
u=\varepsilon A^{\prime}(t)^{-1} \mathcal{A}^{\prime}(t) \delta u+\varepsilon R_{D}^{\prime}(t) \delta \gamma_{0} u+\varepsilon R_{N}^{\prime}(t) \gamma_{1} \frac{\partial_{y}}{t} u
$$

by definition. The notation

$$
[\mathcal{A}(t), \delta]=\mathcal{A}^{\prime}(t) \delta-\delta \mathcal{A}(t)
$$

was used in (3.17). It follows that

$$
\varepsilon\left(\mathcal{A}^{\prime}(t), \mathcal{B}^{\prime}(t)\right)^{-1} \delta(\mathcal{A}(t), \mathcal{B}(t))=\operatorname{id}_{\mathbb{E}_{1}}-\varepsilon A^{\prime}(t)^{-1}[\mathcal{A}(t), \delta]
$$


The term containing the commutator is lower order and can be estimated just like before exploiting the structure of $\mathcal{A}(t)$ in order to yield the invertibility of

$$
\operatorname{id}_{\mathbb{E}_{1}}-\varepsilon A^{\prime}(t)^{-1}[\mathcal{A}(t), \delta]
$$

and, thus, a left inverse for the singular boundary value problem. The result is summarized in the next theorem.

Theorem 3.3. Let $\alpha, \beta \in(0,1), \gamma=0, \beta$ and $0<c_{0} \leq c \in \mathrm{BUC}^{1+\alpha}$. Given

$$
(f, g, h) \in \mathrm{C}_{\gamma}^{\beta} \mathrm{BUC}^{1+\alpha} \mathrm{C} \times \mathrm{C}_{\gamma}^{\beta} \mathrm{BUC}^{3+\alpha} \times \mathrm{C}_{\gamma}^{\beta} \mathrm{BUC}^{2+\alpha}
$$

there exists a unique solution $u$ of (2.1) -(2.3) which belongs to the space

$$
\mathbb{E}_{1}:=\left\{u \in \mathbb{E}_{0} \mid \partial_{x}^{\alpha}\left(\frac{\partial_{y}}{t}\right)^{j} u \in \mathbb{E}_{0} \text { for }|\alpha|, j=0,1,2 \text { s.t. }|\alpha|+j \leq 2\right\}
$$

where

$$
\mathbb{E}_{0}:=\mathrm{C}_{\gamma}^{\beta} \mathrm{BUC}^{1+\alpha} \mathrm{C}
$$

It naturally splits into three components

$$
u=\tilde{A}(t)^{-1} f+\tilde{R}_{N}(t) h+\tilde{R}_{D}(t) g
$$

with the same asymptotic behavior at the origin as in the constant coefficient case and where

$$
\begin{gathered}
\tilde{A}(t)^{-1} f=(\mathcal{A}(t), \mathcal{B}(t))^{-1}(f, 0,0), \tilde{R}_{D}(t) g=(\mathcal{A}(t), \mathcal{B}(t))^{-1}(0, g, 0) \\
\text { and } \tilde{R}_{N}(t) h=(\mathcal{A}(t), \mathcal{B}(t))^{-1}(0,0, h)
\end{gathered}
$$

respectively.

Proof. The only part of the proof missing is for the claim about the asymptotic behavior of the solution. It is obtained using the representation

$$
\left[\begin{array}{ccc}
T & -T[\mathcal{A}(t), \varepsilon] R_{D}^{\prime}(t) \delta & -T[\mathcal{A}(t), \varepsilon] R_{N}^{\prime}(t) \delta \\
0 & \operatorname{id}_{\gamma_{0} \mathbb{E}_{1}} & 0 \\
0 & 0 & \operatorname{id}_{\gamma_{1} \partial_{y} \mathbb{E}_{1}}
\end{array}\right]
$$

for the inverse of

$$
\left[\begin{array}{ccc}
\operatorname{id}_{\mathbb{E}_{0}}-[\mathcal{A}(t), \varepsilon] A^{\prime}(t)^{-1} \delta & {[\mathcal{A}, \varepsilon] R_{D}^{\prime}(t) \delta} & {[\mathcal{A}(t), \varepsilon] R_{N}^{\prime}(t) \delta} \\
0 & \operatorname{id}_{\gamma_{0} \mathbb{E}_{1}} & 0 \\
0 & 0 & \operatorname{id}_{\gamma_{1} \partial_{y} \mathbb{E}_{1}}
\end{array}\right]
$$

where $T=\left[\operatorname{id}_{\mathbb{E}_{0}}-[\mathcal{A}(t), \varepsilon] A^{\prime}(t)^{-1} \delta\right]^{-1}$, the factorization of the resolvent implied by (3.14) and the mapping properties for the operators involved which follow from the symbol analysis combined with Theorem 2.1 Proposition 2.2 and (2.6)-(2.9). Take for instance the entry $T \mathcal{A}(t) \varepsilon R_{D}^{\prime}(t) \delta$. The claim follows from

$$
T \in \mathcal{L}\left(\mathbb{E}_{0}\right), \mathcal{A} \in \mathcal{L}\left(\mathbb{E}_{1}, \mathbb{E}_{0}\right) \text { and } \varepsilon R_{D}^{\prime} \delta \in \mathcal{L}\left(\partial_{D} \mathbb{E}_{1}, \mathbb{E}_{1}\right)
$$

From now on, the tildes on the solution operators of the non-constant coefficient case will be omitted. Since the corresponding operators in the constant coefficient case will no longer be used in the analysis, no confusion seems likely. 
3.3. The Parabolic Problem. It is now possible to return to the analysis of the model problem (2.1)-(2.3) for $\varepsilon=1$. If the boundary conditions are homogeneous, then Theorem 2.3 gives existence and regularity of a solution $u \in \mathbb{E}_{1}$ whenever $f \in \mathbb{E}_{0}$. The notation $\mathbb{E}_{1}$ (see Theorem 3.2 ) has been used so far to describe the regularity space of the singular elliptic boundary value problem. In the parabolic case the same notation indicates the space

$$
\mathbb{E}_{1}:=\left\{u \in \mathbb{E}_{0} \mid \partial_{t} u \in \mathbb{E}_{0}, \partial_{x}^{\alpha}\left(\frac{\partial_{y}}{t}\right)^{j} u \in \mathbb{E}_{0} \text { for }|\alpha|, j=0,1,2 \text { s.t. }|\alpha|+j \leq 2\right\}
$$

in accordance with Theorem 2.3 To obtain optimal regularity results for

$$
A(t)=\triangle_{x}+\frac{1}{t^{2} c^{2}(x)} C
$$

it is therefore sufficient to check that conditions (2.11)-(2.13) are satisfied. The operator $C$ was defined in (3.6). Freezing coefficients arguments such as those in [?, ?] or [?] show that $A(t)$ does indeed generate an analytic semi-group on $E_{0}$ for each fixed $t>0$ which is exponentially decaying since $C$ is invertible (see also the beginning paragraph of Subsection 3.2). Estimates (2.12)-(2.13) follow from the regularity theory developed in the previous section and the fact that $A(t)$ generates an exponentially decaying analytic semi-group. The latter makes it possible, in particular, to define the fractional power appearing in (2.12) (see [?] for more details). Condition (2.13) follows from

$$
\left\|A(t)^{-1}\right\|_{\mathcal{L}\left(\mathbb{E}_{0}\right)} \leq c T^{2}
$$

which, in its turn, follows from the observation that $\left(\frac{1}{c^{2}(x)} C\right)^{-1}$ is bounded in $\mathbb{E}_{0}$ and from

$$
A(t)^{-1}=t^{2}\left(t^{2} \triangle_{x}+\frac{1}{c^{2}(x)} C\right)^{-1} .
$$

The first condition in (2.12) follows from

$$
[A(t)-A(s)] A(\tau)^{-1}=\frac{\left(t^{2}-s^{2}\right) \tau^{2}}{t^{2} s^{2}} \frac{C}{c^{2}(x)}\left[-\tau^{2} \triangle_{x}+\frac{C}{c^{2}(x)}\right]^{-1}
$$

and the fact that

$$
\left\|\frac{C}{c^{2}(x)}\left[-\tau^{2} \triangle_{x}+\frac{C}{c^{2}(x)}\right]^{-1}\right\|_{\mathcal{L}\left(\mathbb{E}_{0}\right)}
$$

is uniformly bounded for $\tau \in(0, T]$. The second condition in (2.12) follows similarly by using abstract mapping properties of fractional powers and, in particular, that

$$
\left\|\left[-\tau^{2} \triangle_{x}+\frac{C}{c^{2}(x)}\right]^{-\rho+1}\right\|_{\mathcal{L}\left(\mathbb{E}_{0}\right)} \leq c .
$$

It remains to be shown that problem (2.1)-(2.3) can be solved for inhomogeneous boundary conditions as well. The result is formulated in the next theorem.

Theorem 3.4. Let $c \in \mathrm{BUC}^{1+\alpha}$ and

$$
f \in \mathbb{E}_{0}, g \in \mathrm{C}_{\beta}^{\beta} \mathrm{BUC}^{3+\alpha} \cap \mathrm{C}_{\beta}^{1+\beta} \mathrm{BUC}^{1+\alpha}, h \in \mathrm{C}_{\beta}^{\beta} \mathrm{BUC}^{2+\alpha} \cap \mathrm{C}_{\beta}^{1+\beta} \mathrm{BUC}^{\alpha} .
$$


Then there exists a unique solution $u \in \mathbb{E}_{1}$ of (2.1) -(2.3).

Proof. It can be assumed without loss of generality that $f \equiv 0$. Looking for a solution $u$ in the form

$$
u=v+R_{D}(t) g+R_{N}(t) h
$$

one obtains that $v$ satisfies

$$
\dot{v}-A(t) v=\frac{d}{d t}\left[R_{D}(t) g+R_{N}(t) h\right] .
$$

Using equations (2.1)-(2.3), it can be checked that

$$
\begin{aligned}
\frac{d}{d t}\left[R_{D}(t) g\right]=\frac{2}{t} A(t)^{-1} \triangle_{x} R_{D}(t) g & +R_{D}(t) \dot{g} \\
\frac{d}{d t}\left[R_{N}(t) h\right] & =\frac{2}{t} A(t)^{-1} \triangle_{x} R_{N}(t) h+\frac{1}{t} R_{N}(t) h+R_{N}(t) \dot{h}
\end{aligned}
$$

The regularity results obtained in the previous section combined with the assumptions then imply that

$$
\left\{t \mapsto \frac{d}{d t}\left[R_{D}(t) g+R_{N}(t) h\right]\right\} \in \mathbb{E}_{0} .
$$

Take for instance $\left[t \mapsto \frac{2}{t} A(t)^{-1} \triangle_{x} R_{D}(t) g\right]$ and observe that

$$
R_{D} \in \mathcal{L}\left(\partial_{D} \mathbb{E}_{1}, \mathbb{E}_{1}\right), \triangle_{x} \in \mathcal{L}\left(\mathbb{E}_{1}, \mathbb{E}_{0}\right) \text { and }\left[t \mapsto \frac{2}{t} A(t)^{-1}\right] \in \mathcal{L}\left(\mathbb{E}_{0}\right)
$$

or $R_{D}(t) \dot{g}$ in which case the stated regularity follows from

$$
\dot{g} \in \gamma_{0} \mathbb{E}_{0} \text { and } R_{D} \in \mathcal{L}\left(\gamma_{0} \mathbb{E}_{0}, \mathbb{E}_{0}\right) .
$$

The fact that $R_{D} \in \mathcal{L}\left(\gamma_{0} \mathbb{E}_{0}, \mathbb{E}_{0}\right)$ has not been explicitly proven but can be obtained by symbol analysis and freezing coefficients along the lines of Sections 3.1 and 3.2. The claim then follows from Theorem $2.3 \mathrm{Q}$

4. The Hamilton-Jacobi Equation. Consider the Hamilton-Jacobi equation

$$
\begin{aligned}
s_{t}-\sqrt{1+|\nabla s|^{2}} v & =0 \text { in }(0, \infty) \times \mathbb{R}^{n}, \\
s(0, \cdot) & \equiv 0 \text { on } \mathbb{R}^{n}
\end{aligned}
$$

for a given $v \in \mathrm{C}^{\beta} \mathrm{BUC}^{3+\alpha}$ satisfying $v(0, \cdot)=g$. The method of characteristics allows one to recast this Hamilton-Jacobi equation as a system of ODEs in the following manner

$$
\begin{array}{ll}
\dot{t}=1, & t(0)=0, \\
\dot{x}=-\frac{p}{\sqrt{1+|p|^{2}}} v(t, x), & x(0)=\rho \in \mathbb{R}^{n}, \\
\dot{z}=r-\frac{|p|^{2}}{\sqrt{1+|p|^{2}}} v(t, x), & z(0)=0, \\
\dot{r}=\sqrt{1+|p|^{2}} \partial_{t} v(t, x), & r(0)=g(\rho) \\
\dot{p}=\sqrt{1+|p|^{2}} \nabla_{x} v(t, x), & p(0)=0 .
\end{array}
$$


This system is easily seen to reduce to

$$
\begin{aligned}
& \dot{x}=-\frac{p}{\sqrt{1+|p|^{2}}} v(t, x), x(0)=\rho \in \mathbb{R}^{n}, \\
& \dot{p}=\sqrt{1+|p|^{2}} \nabla_{x} v(t, x), p(0)=0
\end{aligned}
$$

as all other unknowns can be obtained after solving this reduced system. The assumption on $v$ makes it possible to solve this system on a possibly small time interval $[0, T]$ which is independent of $\rho \in \mathbb{R}^{n}$. Exploiting the regularity assumption on $v$ it can easily be seen that the flow mapping

$$
\left(X_{t}, P_{t}\right): \mathbb{R}^{2 n} \rightarrow \mathbb{R}^{2 n},(\rho, \eta) \mapsto(x(t, \rho, \eta), p(t, \rho, \eta)),
$$

satisfies

$$
\left[t \mapsto\left(X_{t}, P_{t}\right)\right] \in \mathrm{C}^{\beta} \mathrm{BUC}^{2+\alpha}\left(\mathbb{R}^{2 n}, \mathbb{R}^{2 n}\right)
$$

where the map is obtained by solving system (4.8)-(4.9) with the second initial condition substituted by $p(0)=\eta$. It is clearly a flow of diffeomorphisms. Furthermore, since an equation satisfied by $\left(D_{\rho} x, D_{\rho} p\right)$ is easily derived from (4.8)-(4.9) and since

$$
\left(D_{\rho} x(0, \rho, \eta), D_{\rho} p(0, \rho, \eta)\right)=\left(\operatorname{id}_{\mathbb{R}^{n}}, 0\right),
$$

it follows that $D_{\rho} X_{t}(\rho, 0)$ satisfies

$$
\left\|\operatorname{id}_{\mathbb{R}^{n}}-D_{\rho} X_{t}(\rho, 0)\right\| \leq \frac{1}{2}
$$

uniformly in $\rho \in \mathbb{R}^{n}$. This implies that $X_{t}(\cdot, 0)$ is a diffeomorphism and yields uniform estimates for the inverse of $X_{t}$. It follows that

$$
\left[t \mapsto\left(X_{t}\right)^{*}\right],\left[t \mapsto\left(X_{t}\right)_{*}\right] \in \mathrm{C}^{\beta} \mathcal{L}\left(\mathrm{BUC}^{2+\alpha}\right)
$$

where we denoted the pull-back and push-forward with $X_{t}$ by $\left(X_{t}\right)^{*}$ and $\left(X_{t}\right)_{*}$, respectively. Moreover, these operators are uniformly bounded in norm in a small time interval.

Theorem 4.1. Assume that $v \in \mathrm{C}^{\beta} \mathrm{BUC}^{3+\alpha}$ be given with $v(0)=g \in \mathrm{BUC}^{3+\alpha}$. Then, for $T>0$ small enough, there exists a unique solution $s$ of (4.1)-(4.2) on $[0, T]$ with

$$
s \in \mathrm{C}^{1+\beta} \mathrm{BUC}^{2+\alpha} \cap \mathrm{C}^{\beta} \mathrm{BUC}^{3+\alpha} .
$$

The existence interval is independent of $v$ in a neighborhood of $g$. Furthermore, if $v_{1}, v_{2} \in \mathrm{C}^{\beta} \mathrm{BUC}^{3+\alpha}$ are such that $v_{1}(0)=v_{2}(0)=g$, then

$$
\begin{aligned}
\left\|s_{1}-s_{2}\right\|_{\mathrm{C}^{1+\beta} \mathrm{BUC}^{2+\alpha} \cap \mathrm{C}^{\beta} \mathrm{BUC}^{3+\alpha}} & \\
& \leq c T\left\|v_{1}-v_{2}\right\|_{\mathrm{C}^{\beta} \mathrm{BUC}^{3+\alpha}}+c\left\|v_{1}-v_{2}\right\|_{\mathrm{C}^{\beta} \mathrm{BUC}^{2+\alpha}} .
\end{aligned}
$$

Proof. The solution can be computed by solving the reduced system of characteristic equations (4.8)-(4.9), which produces in particular the diffeomorphisms $X_{t}$. 
A minimal interval of existence can be chosen independently of the initial conditions thanks to the assumptions on $v$. Next

$$
\nabla_{x} s(t, x)=p\left(t, X_{t}^{-1}(x), 0\right)
$$

shows together with the discussion preceding the formulation of the theorem, in particular (4.10), that

$$
s \in \mathrm{C}^{\beta} \mathrm{BUC}^{3+\alpha} .
$$

The desired time regularity has to be backed out from equations (4.5)-(4.6). The time derivative appearing in the right-hand-side of (4.6) is obviously not welcome in view of the assumptions made on $v$. It is, however, possible to rewrite the term containing said time derivative while integrating the equation to read

$$
\begin{aligned}
& r(t, x)=\sqrt{1+|p(t, x)|^{2}} v(t, x)+ \\
& -\int_{0}^{t}\left[v \nabla_{x} v \cdot p\right]\left(\tau, X_{\tau}\left(X_{t}^{-1}(x)\right)\right) d \tau-\int_{0}^{t}\left[\frac{|p|^{2}}{\sqrt{1+|p|^{2}}} v\right]\left(\tau, X_{\tau}\left(X_{t}^{-1}(x)\right)\right) d \tau .
\end{aligned}
$$

The solution $s$ can then be obtained by one further integration from (4.5). By (4.10) and the regularity assumption on $v$ it then follows that

$$
s \in \mathrm{C}^{1+\beta} \mathrm{BUC}^{2+\alpha} .
$$

The use of this argument needs to be justified. This can be done by substituting $v$ by a regularized version of it which is differentiable in time and for which the partial integration used to obtain (4.12) can safely be performed. Letting it converge back to the original function $v$ produces a function satisfying the modified equation. Since the solution of the original problem can be constructed by solving only the reduced problem (4.8)-(4.9) no trouble is encountered in taking the limit.

The additional estimate follows from the characteristic system (4.3)-(4.7), the regularity assumptions on $v_{1}, v_{2}$ and the fact that $v_{1}(0)-v_{2}(0)=0$.

5. Existence Result. The local existence result for regular solutions of (1.1)(1.5) is based on the analysis of (1.6)-(1.10). The regularity theory for linear singular elliptic and parabolic problems described in the previous sections and the analysis for the Hamilton-Jacobi equation performed in the last section will be the main tools. It should be kept in mind that $\varepsilon=1$ without loss of generality. To better highlight the structure of the problem and the use of the linear regularity theory it is convenient to rewrite (1.6)-(1.8) using (1.9) in the following form

$$
\begin{aligned}
\dot{u}-\widetilde{\mathcal{A}}(t) u & =\overline{\mathcal{A}}(s) u & & \text { in } S \\
u & =g & & \text { on } \Gamma_{0} \\
\frac{1}{t} \partial_{y} u & =H(s, u) & & \text { on } \Gamma_{1}
\end{aligned}
$$

where $\widetilde{\mathcal{A}}(t)=\triangle_{x}+\frac{1}{t^{2} g^{2}(x)} \partial_{y}^{2}+\frac{y}{t} \partial_{y}$ and the operator $\overline{\mathcal{A}}(s)$ is defined by

$$
\begin{aligned}
\overline{\mathcal{A}}(s) u=\left[\frac{1+y^{2}|\nabla s|^{2}}{s^{2}}-\frac{1}{t^{2} g^{2}(x)}\right] \partial_{y}^{2} u+y\left[\frac{\dot{s}}{s}-\frac{1}{t}\right] \partial_{y} u-2 y \frac{1}{s}\left(\nabla s \mid \partial_{y} \nabla u\right) & \\
& -y \frac{s \triangle s-2|\nabla s|^{2}}{s^{2}} \partial_{y} u
\end{aligned}
$$


and $H(s, u)$ is given by

$$
H(s, u)=\frac{s}{t} \frac{1}{1+|\nabla s|^{2}}(\nabla s \mid \nabla u)-\frac{s}{t} \frac{1}{\sqrt{1+|\nabla s|^{2}}} u(1+u),
$$

respectively.

REMARK 5.1. It should be observed that the operator family $\widetilde{\mathcal{A}}(t)$ does not coincide with the one considered in the sections devoted to elliptic and parabolic regularity theory and denoted by $A(t)$. It is, however, easy to check that is enjoys the same "singular" elliptic regularity theory as a perturbation argument shows

$$
\widetilde{A}(t)=A(t)\left[\operatorname{id}_{\mathbb{E}_{1}}+A^{-1}(t) \frac{y}{t} \partial_{y}\right]=\left[\operatorname{id}_{\mathbb{E}_{0}}+\frac{y}{t} \partial_{y} A^{-1}(t)\right] A(t)
$$

Associated with the new family, there will be boundary solution operators $\widetilde{R}_{D}(t)$ and $\widetilde{R}_{N}(t)$ with corresponding mapping properties. These new boundary operators are actually given by

$$
\widetilde{R}_{D}(t)=R_{D}(t)+\widetilde{A}(t)^{-1}\left[\frac{y}{t} \partial_{y} R_{D}(t)\right] \text { and } \widetilde{R}_{N}(t)=R_{N}(t)+\widetilde{A}(t)^{-1}\left[\frac{y}{t} \partial_{y} R_{N}(t)\right],
$$

where $R_{D}$ and $R_{N}$ are the original boundary operators. From now on the old notation will apply to the new operators.

Assume now that $s \in \mathbb{S}$, that is, that $s \in \mathrm{C}^{1+\beta} \mathrm{BUC}^{2+\alpha} \cap \mathrm{C}^{\beta} \mathrm{BUC}^{3+\alpha}$ is chosen in a small ball about the function $[t \mapsto t g(x)]$ (in the natural norm of $\mathbb{S}$ ) and satisfying

$$
s(0)=0, \dot{s}(0)=g .
$$

It can be verified that the operator $\overline{\mathcal{A}}(s) \in \mathcal{L}\left(\mathbb{E}_{1}, \mathbb{E}_{0}\right)$ is small in the operator norm if the time interval length $T>0$ is chosen small (this the reason why the modified operator $\widetilde{A}(t)$ is introduced, see Remark 5.1). This structure will be useful in the proof of the following existence result.

Theorem 5.1. Let $s \in \mathbb{S}$ be given with the above properties (5.4), $g \in \mathrm{BUC}^{4+\alpha}$, and let $T>0$ be small. Then there exists a unique solution $u \in \mathbb{E}_{1}$ of (5.1)-(5.3) which is the fixed-point of the operator

$$
\Phi_{1}(u)=v+R_{D}(t) g+R_{N}(t) H(s, u)
$$

where $v$ solves

$$
\dot{v}-A(t) v=\overline{\mathcal{A}}(s) u-\frac{2}{t} A^{-1}(t)\left[\triangle_{x}+\frac{y}{t} \partial_{y}\right]\left[R_{D}(t) g+R_{N}(t) H(s, u)\right]
$$

Moreover, for $s_{1}, s_{2} \in \mathbb{S}$, the following estimates

$$
\begin{aligned}
& \left\|\gamma_{1} u_{1}-\gamma_{1} u_{2}\right\|_{\mathrm{C}^{\beta} \mathrm{BUC}^{3+\beta}} \leq c\left\|s_{1}-s_{2}\right\|_{\mathbb{S}^{\beta}} \\
& \left\|\gamma_{1} u_{1}-\gamma_{1} u_{2}\right\|_{\mathrm{C}^{\beta} \mathrm{BUC}^{2+\beta}} \leq c T^{\beta}\left\|s_{1}-s_{2}\right\|_{\mathbb{S}^{\beta}}
\end{aligned}
$$

hold.

Proof. As already pointed, the existence proof relies on maximal regularity results obtained in the previous sections which are at the heart of the matter and make it now possible to use a simple Banach fixed-point argument. The fixed-point $u$ will be looked for in the set $\left\{u \in \overline{\mathbb{B}}_{\mathbb{E}_{1}}(g, r) \mid u(0)=g\right\}$ for positive $r$ to be fixed later. This set 
is endowed with the $\mathbb{E}_{1}$ topology and is therefore complete. It is easy but tedious to show that $v, R_{N}(t) H(s, u)$ are in $\mathbb{E}_{1} \cap \mathbb{E}_{0,0}$. The first regularity claim follows directly from Theorems 3.3 and 3.4 both for $v$ and $R_{N}(t) H(s, u)$. The vanishing property for $v$ follows from its regularity using the equations it satisfies. Its regularity readily implies that $\partial_{y}^{2} v(t=0) \equiv 0$. Since $v$ satisfies homogeneous boundary conditions

$$
v(y=0)=0=\partial_{y} v(y=1)
$$

it then is $v(t=0) \equiv 0$. As for the boundary term $R_{N}(t) H(s, u)$ it follows from

$$
\frac{1}{t} R_{N}(t) \in \mathcal{L}\left(\mathrm{C}_{0}^{\beta} \mathrm{BUC}^{1+\alpha}, \mathbb{E}_{0,0}\right)
$$

combined with

$$
t H(s, u) \in \mathrm{C}_{0}^{\beta} \mathrm{BUC}^{1+\alpha},
$$

or, alternatively, also from the equations. Finally the equations also imply that

$$
\partial_{y}^{2} R_{D}(0) g \equiv 0, R_{D}(0) g(y=0)=g, \partial_{y} R_{D}(0) g(y=1)=0 .
$$

It clearly follows that $R_{D}(0) g \equiv g$. Summarizing it is obtained that $\Phi_{1}(u)(0)=g$. Next a norm estimate for $\Phi_{1}(u)-g$ is needed. The term $R_{D}(t) g-g$ can be estimated by some fixed constant $r_{1}$. As for the other boundary term one has that

$$
\left\|\frac{R_{N}(t)}{t}[t H(s, u)]\right\|_{\mathbb{E}_{1}} \leq c r(1+r) T^{\beta}\|s\|_{\mathbb{S}}
$$

and can therefore be made arbitrarily small by reducing the interval length. The above estimate is possible since the map $[t \mapsto t H(s, u)]$ vanishes at the origin in view of the properties of $s$. Next $v$ has to be estimated. Most terms can simply be estimated in a way that they produce a factor $T^{\beta}$ and can be made arbitrarily small by interval length reduction. This is due to the fact that

$$
\|v\|_{\mathbb{E}_{1}} \leq\left\|\left(\partial_{t}-A(t)\right)^{-1}\right\|_{\mathcal{L}\left(\mathbb{E}_{0}, \mathbb{E}_{1}\right)}\|F(s, u)\|_{\mathbb{E}_{0}}
$$

where $F(s, u)$ summarizes the terms in the right-hand-side of (5.5), and the specifics of $F$. Consider for instance the first term $\overline{\mathcal{A}}(s) u$. Since $\overline{\mathcal{A}}(s)$ vanishes at the origin, it can be seen that

$$
\|\overline{\mathcal{A}}(s)\|_{\mathcal{L}\left(\mathbb{E}_{1}, \mathbb{E}_{0}\right)} \leq c T^{\beta}\|\dot{s}\|_{\mathcal{S}}
$$

which implies the claimed estimate. The estimate for the second term follows from the fact that

$$
\left\|\frac{1}{t} A(t)^{-1}\right\|_{\mathcal{L}\left(\mathbb{E}_{0}\right)} \leq c T
$$

and the general mapping properties of the operators involved. All remaining terms but one can be handled similarly. All terms in $\frac{d}{d t}[t H(s, u)]$ containing either $s$ or $\nabla s$ are going to lead to more terms vanishing at the origin. The term

$$
\frac{R_{N}(t)}{t}\left[\frac{\dot{s}}{\sqrt{1+|\nabla s|^{2}}} u(1+u)\right]
$$


behaves slightly differently and needs separate consideration since $\dot{s} u(1+u) / \sqrt{1+|\nabla s|^{2}}$ does not vanish at the origin. Rewriting this term as

$$
\frac{R_{N}(t)}{t}\left[\frac{\dot{s}}{\sqrt{1+|\nabla s|^{2}}} u(1+u)-g^{2}(1+g)\right]+\frac{R_{N}(t)}{t}\left[g^{2}(1+g)\right]
$$

and using that $u(0)=g$ up to vanishing terms of type $T^{\beta}$, it follows that

$$
\left\|\frac{R_{N}(t)}{t}\left[\frac{\dot{s}}{\sqrt{1+|\nabla s|^{2}}} u(1+u)\right]\right\|_{\mathbb{E}_{0}} \leq r_{2}+c r^{2} T^{\beta}\|s\|_{\mathbb{S}}
$$

where the constant $r_{2}$ is given by

$$
r_{2}=\left\|\left(\partial_{t}-A(t)\right)^{-1} \frac{R_{N}(t)}{t}\left[g^{2}(1+g)\right]\right\|_{\mathbb{E}_{1}} .
$$

It follows that $\Phi_{1}$ is self-map if $r$ is chosen to satisfy $r>r_{1}+r_{2}$ and the time interval length is sufficiently small. It is important to observe that all estimates have constants which are independent of $s$ in the chosen neighborhood.

Next it needs to be shown that $\Phi_{1}$ is a contraction. The calculations are very similar to the above. The following terms

$$
\Phi\left(u_{1}\right)-\Phi_{1}\left(u_{2}\right)=v_{1}-v_{2}+R_{N}(t)\left[H\left(s, u_{1}\right)-H\left(s, u_{2}\right)\right]
$$

need to be estimated. The difference $v_{1}-v_{2}$ satisfies

$$
v_{1}-v_{2}=\left(\partial_{t}-A(t)\right)^{-1}\left[F\left(s, u_{1}\right)-F\left(s, u_{2}\right)\right] .
$$

Maximal regularity yields that

$$
\left\|v_{1}-v_{2}\right\|_{\mathbb{E}_{1}}=\left\|\left(\partial_{t}-A(t)\right)^{-1}\left[F\left(s, u_{1}\right)-F\left(s, u_{2}\right)\right]\right\|_{\mathbb{E}_{1}} \leq c\left\|F\left(s, u_{1}\right)-F\left(s, u_{2}\right)\right\|_{\mathbb{E}_{0}}
$$

so that only the last term needs to be estimated. As for the other summand, an estimate for

$$
\left\|R_{N}(t)\left[H\left(s, u_{1}\right)-H\left(s, u_{2}\right)\right]\right\|_{\mathbb{E}_{1}}
$$

needs to be established. Using similar estimates as for the self-map property and observing that all the terms not vanishing at the origin drop out since they do not depend on the unknowns one arrives at

$$
\left\|\Phi_{1}\left(s, u_{1}\right)-\Phi_{1}\left(s, u_{2}\right)\right\|_{\mathbb{E}_{1}} \leq c T^{\beta}\left\|u_{1}-u_{2}\right\|_{\mathbb{E}_{1}}
$$

with a constant which is independent of $s$ in the chosen neighborhood. Existence of a unique fixed-point follows by making the interval length short enough.

Next observe that any solution actually satisfies

$$
\gamma_{1} u=\gamma_{1} R_{D}(t) g+\gamma_{1}\left[v+R_{N}(t) H(s, u)\right] \in \mathrm{C}^{\beta} \mathrm{BUC}^{3+\alpha}
$$

by Remark 2.1 since $g \in \mathrm{BUC}^{4+\alpha}$. This follows from

$$
v=\left(\partial_{t}-A(t)\right)^{-1}\left[F(s, u)+\frac{1}{t} R_{N}(t) g^{2}(1+g)\right]-\left(\partial_{t}-A(t)\right)^{-1} \frac{1}{t} R_{N}(t) g^{2}(1+g)
$$


and

$$
R_{N}(t) H(s, u)=R_{N}(t)\left[H(s, u)-g^{2}(1+g)\right]+R_{N}(t) g^{2}(1+g),
$$

where the first terms on the right-hand-sides above belong to $\mathbb{E}_{1,0}$ and the second ones are not singular due to the regularity of $g$, and therefore of $g^{2}(1+g)$, combined with Remark 2.1 The first continuous dependence result follows along the lines of the above estimates from the fact that the the solution to the initial boundary value problem depends smoothly (linearly) on the interior and boundary data $\overline{\mathcal{A}}(s) u$ and $H(s, u)$. The only difference stems from the fact that a stronger norm is now estimated which leads to the absence of the factor $T^{\beta}$ in front of $\left\|s_{1}-s_{2}\right\|_{\mathbb{S}}$. This factor can be regained if a weaker norm is estimated in view of the "desingularizing" properties of $\left[\partial_{t}-A(t)\right]^{-1}$, which leads to the stated continuous dependence estimate. The estimates are based on

$$
\begin{gathered}
\left\|\gamma_{1} u_{1}-\gamma_{1} u_{2}\right\|_{\mathrm{C}^{\beta} \mathrm{BUC}^{3+\beta}} \leq\left\|\gamma_{1} \Phi_{1}\left(s_{1}, u_{1}\right)-\gamma_{1} \Phi_{1}\left(s_{2}, u_{2}\right)\right\|_{\mathrm{C}^{\beta} \mathrm{BUC}^{3+\beta}} \\
\leq \| \gamma_{1} \Phi_{1}\left(s_{1}, u_{1}\right)- \\
\gamma_{1} \Phi_{1}\left(s_{1}, u_{2}\right) \|_{\mathrm{C}^{\beta} \mathrm{BUC}^{3+\beta}} \\
+\left\|\gamma_{1} \Phi_{1}\left(s_{1}, u_{2}\right)-\gamma_{1} \Phi_{1}\left(s_{2}, u_{2}\right)\right\|_{\mathrm{C}^{\beta} \mathrm{BUC}^{3+\beta}}
\end{gathered}
$$

The estimate for the first term of $\overline{\mathcal{A}}\left(s_{1}\right)-\overline{\mathcal{A}}\left(s_{2}\right)$ is typical. It gives

$$
\begin{aligned}
& \left\|\left[\frac{t^{2}}{s_{1}^{2}}\left(1+y^{2}\left|\nabla s_{1}\right|^{2}\right)-\frac{t^{2}}{s_{2}^{2}}\left(1+y^{2}\left|\nabla s_{2}\right|^{2}\right)\right] \frac{\partial_{y}^{2}}{t^{2}}\right\|_{\mathcal{L}\left(\mathbb{E}_{1}, \mathbb{E}_{0,0}\right)} \\
& \leq\left\|\frac{t^{2}}{s_{1}^{2}}\left(1+y^{2}\left|\nabla s_{1}\right|^{2}\right)-\frac{t^{2}}{s_{2}^{2}}\left(1+y^{2}\left|\nabla s_{2}\right|^{2}\right)\right\|_{\mathrm{C}^{\beta} \mathrm{BUC}^{1+\alpha} \mathrm{C}}\left\|\frac{\partial_{y}^{2}}{t^{2}}\right\|_{\mathcal{L}\left(\mathbb{E}_{1}, \mathbb{E}_{0}\right)}
\end{aligned}
$$

which easily entails the claim since

$$
\begin{aligned}
\| \frac{t^{2}}{s_{1}^{2}}\left(1+y^{2}\left|\nabla s_{1}\right|^{2}\right) & -\frac{t^{2}}{s_{2}^{2}}\left(1+y^{2}\left|\nabla s_{2}\right|^{2} \|_{\mathrm{C}^{\beta} \mathrm{BUC}^{1+\alpha} \mathrm{C}}\right. \\
& \leq c\left(\left\|\dot{s}_{1}-\dot{s}_{2}\right\|_{\mathrm{C}^{\beta} \mathrm{BUC}^{1+\alpha}}+\left\|s_{1}-s_{2}\right\|_{\mathrm{C}^{\beta} \mathrm{BUC}^{2+\alpha}}\right) \leq c\left\|s_{1}-s_{2}\right\|_{\mathcal{S}} .
\end{aligned}
$$

One is eventually lead to

$$
\left\|\gamma_{1} u_{1}-\gamma_{1} u_{2}\right\|_{\mathrm{C}^{\beta} \mathrm{BUC}^{3+\alpha}} \leq c T^{\beta}\left\|u_{1}-u_{2}\right\|_{\mathbb{E}_{1}}+\left\|s_{1}-s_{2}\right\|_{\mathcal{S}^{\beta}}
$$

from which the claim follows since the first term on the right can be absorbed in the left-hand-side.

All pieces are now in place in order to show existence for the original system of equations (1.6)-(1.10). By denoting with $u=\Phi_{1}(s)$ the solution of (1.6)-(1.8) for a given $s \in \mathcal{S}$, it follows that

$$
u \in \mathbb{E}_{1} \text { and } \gamma_{1} u \in \mathrm{C}^{\beta} \mathrm{BUC}^{3+\alpha} .
$$

Decomposition (5.7) shows that $u$ is in a given neighborhood of $g$ for any choice of $s \in \mathcal{S}$ in a neighborhood of $[t \mapsto t g(x)]$. Similarly let $\Phi_{2}(u)$ be the solution of (1.9)-(1.10) constructed in section 4 In this case, if $u \in \mathbb{E}_{1}$ with $\gamma_{1} u \in \mathrm{C}^{\beta} \mathrm{BUC}^{3+\alpha}$, $\Phi_{2}(u) \in \mathcal{S}$ will be in a neighborhood of $\operatorname{tg}(x)$ for $u$ in a neighborhood of $g$. It follows that a solution to the full problem can be found by producing a fixed-point $s$ for the map $\Phi:=\Phi_{2} \circ \Phi_{1}$ and defining $u:=\Phi_{1}(s)$. Summarizing 
Theorem 5.2. For any given $g \in \mathrm{BUC}^{4+\alpha}$ such that $g(x) \geq g_{0}>0, x \in \mathbb{R}^{n}$, there exists a unique local solution $(u, s)$ of the free boundary problem (1.6) (1.10) such that

$$
\begin{aligned}
& u, \dot{u}, \partial_{x}^{\alpha} \frac{1}{t^{k}} \partial_{y}^{k} u \in \mathrm{C}_{\beta}^{\beta} \mathrm{BUC}^{1+\alpha} \mathrm{C} \text { for } 0 \leq|\alpha|+k \leq 2 \\
& \qquad \gamma_{1} u \in \mathrm{C}^{\beta} \mathrm{BUC}^{3+\alpha} \text { and } \\
& \qquad s \in \mathrm{C}^{1+\beta} \mathrm{BUC}^{2+\alpha} \cap \mathrm{C}^{\beta} \mathrm{BUC}^{3+\alpha} .
\end{aligned}
$$

Furthermore

$$
u=R_{D}(t) g+R_{N}(t) H(s, u)+v
$$

where $v$ is a solution of (5.5) and the different terms have different asymptotic behavior in the origin. They behave respectively like $t^{k}$ for $k=0,1,2$ modulo the singular behavior built-in in the space chosen.

Proof. Making the time interval as small as needed and choosing $r>0$ large enough it can be seen that $\left(\Phi_{1}, \Phi_{2}\right)$ is a self-map on the complete set

$$
\left\{u \in \overline{\mathbb{B}}_{\mathbb{E}_{1}}(0, r) \mid u(0)=g\right\} \times\left\{s \in \overline{\mathbb{B}}_{\mathbb{S}}\left(g t, g_{0} / 2\right) \mid s(0)=0, \dot{s}(0)=g\right\} .
$$

where the additional requirements $u(0) \equiv g, s(0)=0, \dot{s}(0) \equiv g$ are included in the definition of the balls. Combining the estimates of Theorems 4.1] and 5.1] it follows that

$$
\left\|\Phi\left(s_{1}\right)-\Phi\left(s_{2}\right)\right\|_{\mathbb{S}^{\beta}} \leq c T^{\beta}\left\|s_{1}-s_{2}\right\|_{\mathbb{S}^{\beta}}
$$

and the contraction principle can be applied to obtain existence.

REMARK 5.2. It should be pointed out that this is the first well-posedness result for this class of singular free boundary problems in more than one space dimension for the full evolutionary problem. A companion result for the quasi-stationary approximation $(\varepsilon=0)$ has previously been obtained in [?]. 